\title{
Nematodiasis gastrointestinal en caprinos de Ica, Perú
}

\author{
Gastrointestinal nematodiasis in goats from Ica, Peru \\ María Cáceres V. ${ }^{1}$, Rosa Ysabel Pinedo V. ${ }^{1}$, Amanda Chávez V. ${ }^{1,2}$
}

\section{Resumen}

\begin{abstract}
El objetivo del estudio fue determinar la prevalencia de nematodos gastrointestinales en caprinos de cuatro distritos del departamento de Ica, Perú, así como establecer la asociación entre la prevalencia de estos parásitos con las variables edad, sexo y procedencia, determinar el promedio de carga parasitaria e identificar los géneros y especies de los nematodos presentes. Se colectaron muestras de heces de 728 cabras de los distritos de Independencia, Humay, El Carmen y Chincha Baja entre junio y agosto de 2017. Para la identificación y la evaluación de la carga parasitaria se emplearon las técnicas de flotación y McMaster Modificado, respectivamente. Además, se utilizaron las técnicas de Corticelli y Lai Modificado y Baermann para el cultivo e identificación de las larvas infectivas (L3). Se obtuvo una prevalencia general de nematodos gastrointestinales de 67.6\% (IC 95\%: 64.1-70.9), siendo de 92.5, 80.8, 59.1, 41.9\% en los distritos de Independencia, Humay, El Carmen y Chincha Baja, respectivamente. Se encontró asociación significativa con todas las variables en estudio $(\mathrm{p}<0.05)$. Se determinó una prevalencia de 63.7, 12.9 y $4.1 \%$ para los huevos tipo Strongylus (HTS), Skrjabinema spp y Trichuris spp, respectivamente. El promedio de la media geométrica general de la carga parasitaria fue de HTS (259.4 hpg), Skrjabinema spp (70 hpg) y Trichuris spp (54.3 hpg), consideradas como cargas bajas. Los géneros y especies de lavas infectivas L3 cultivadas de huevos HTS fueron: Haemonchus spp (45\%), Trichostrongylus spp (37\%), Ostertagia spp (15\%), Chabertia ovina (0.6\%) y Cooperia spp (0.6\%).
\end{abstract}

Palabras clave: cabras, nematodos, Ica, huevo tipo Strongylus

${ }^{1}$ Laboratorio de Microbiología y Parasitología Veterinaria, Facultad de Medicina Veterinaria, Universidad Nacional Mayor de San Marcos, Lima, Perú

2E-mail: achavezv@unmsm.edu.pe

Recibido: 1 de febrero de 2021

Aceptado para publicación: 20 de agosto de 2021

Publicado: 27 de octubre de 2021

CLos autores. Este artículo es publicado por la Rev Inv Vet Perú de la Facultad de Medicina Veterinaria, Universidad Nacional Mayor de San Marcos. Este es un artículo de acceso abierto, distribuido bajo los términos de la licencia Creative Commons Atribución 4.0 Internacional (CC BY 4.0) [https:// creativecommons.org/licenses/by/4.0/deed.es] que permite el uso, distribución y reproducción en cualquier medio, siempre que la obra original sea debidamente citada de su fuente original 
The aim of this study was to determine the prevalence of gastrointestinal nematodes in goats from four districts of the department of Ica, Peru, as well as to establish the association between the prevalence of these parasites with the variables age, sex and origin, determine the average parasite load and identify the genera and species of nematodes present. Stool samples were collected from 728 goats from the districts of Independencia, Humay, El Carmen and Chincha Baja between June and August 2017. For the identification and evaluation of the parasite load, the floatation techniques and modified McMaster were used. In addition, the techniques of Corticelli and modified Lai and Baermann were used for the culture and identification of the infective larvae (L3). A general prevalence of gastrointestinal nematodes of $67.6 \%$ (95\% CI: 64.1-70.9) was obtained, being $92.5,80.8,59.1,41.9 \%$ in the districts of Independencia, Humay, El Carmen and Chincha Baja, respectively. A significant association was found with all the variables under study $(\mathrm{p}<0.05)$. A prevalence of $63.7,12.9$ and $4.1 \%$ was determined for Strongylus (HTS), Skrjabinema spp and Trichuris spp eggs, respectively. The general geometric mean of the parasite load was HTS (259.4 hpg), Skrjabinema spp (70 hpg) and Trichuris spp (54.3 hpg), considered as low loads. The genera and species of infective lavas L3 cultured from HTS eggs were: Haemonchus spp (45\%), Trichostrongylus spp (37\%), Ostertagia spp (15\%), Chabertia ovina (0.6\%) and Cooperia spp (0.6\%).

Key words: goats, nematodes, Ica, Strongylus type egg

\section{INTRODUCCIÓN}

La crianza del ganado caprino es de gran importancia por su contribución en la economía de los pequeños productores agropecuarios de bajos recursos (Boyazoglu et al., 2005; Hassan et al., 2011), debido a que se alimentan de rastrojos de cosecha y pastos naturales generando bajos costos de producción (Arroyo, 2007; Odogu y Okaka, 2016; MIDAGRI, 2017). A diferencia del ganado bovino y ovino, pueden sobrevivir en ambientes agrestes y secos, son más resistentes a enfermedades y sus productos (carne y leche) tienen alto valor nutricional (Flores et al., 2009). Así, la leche de cabra posee alta digestibilidad, altos niveles de ácidos grasos de cadena corta y mediana, calcio, fosforo, potasio, magnesio, vitaminas A y D y es sustituto ideal de la leche de vaca en pacientes alérgicos (Park y Haenlein, 2006; Flores et al., 2009).
En el Perú, la crianza de ganado caprino está principalmente distribuida en la costa y sierra, especialmente en las regiones Piura, Ayacucho, Ancash, Lima e Ica (Arroyo, 2007; MIDAGRI, 2015). Según el IV Censo Nacional Agropecuario (INEI, 2012), el departamento de Ica posee una población caprina de 72112 animales, ocupando el quinto lugar con respecto a la población nacional. Asimismo, 39.2 y $38.3 \%$ de esta población pertenecen a las provincias de Pisco y Chincha, respectivamente (INEI, 2012).

La nematodiasis gastrointestinal en el caprino tiene gran importancia económica debido a que afecta su crecimiento y producción (Cordero del Campillo et al., 1999; Sukupayo y Rayamajhee, 2018), pues afecta entre otros aspectos la conversión alimenticia generando mermas en la producción de carne y leche (Quiroz, 2008; Odogu y Okaka, 2016). Los géneros más frecuentes que se han reportado en caprinos son: Haemonchus, 
Trichostrongylus, Ostertagia, Cooperia, Nematodirus, Oesophagostomum y las especies Trichuris ovis y Skjabinema caprae (Barriga, 2002; Sukupayo y Rayamajhee, 2018). La sintomatología clínica varía según la especie parasitaria predominante, estado nutricional, grado de infección y la edad del animal, pero generalmente se manifiesta con anemia, hipoproteinemia, ascitis, edema submandibular, desnutrición y diarreas intermitentes (Quiroz, 2008; Odogu y Okaka, 2016).

No se dispone de estudios recientes realizados en el país sobre el parasitismo gastrointestinal en caprinos y muchos de ellos fueron realizados hace más de 25 años, siendo mayormente hallazgos de necropsia (Chávez y Guerrero, 1960; Carruitero, 1966; Gonzales, 1968; Nolte, 1979; Casas, 1993). En dichos estudios se encontró prevalencias altas en cabras de crianza extensivas, reportándose $97 \%$ en la provincia de Yauyos (Torres, 1952) y 69\% en Cañete (Casas, 1993). El trabajo más reciente fue realizado por Zárate et al. (2017) en Canta, utilizando la técnica de FAMACHA como indicador del grado de anemia en casos de haemonchosis.

El objetivo principal del presente estudio fue determinar la prevalencia de nematodos gastrointestinales en caprinos de cuatro distritos del departamento de Ica. Asimismo, establecer la asociación entre la prevalencia de nematodos gastrointestinales con las variables edad, sexo y procedencia, determinar el promedio de la carga parasitaria e identificar los géneros y especies de nematodos presentes.

\section{Materiales y Métodos}

\section{Lugar de Estudio}

El estudio se realizó en cuatro distritos del departamento de Ica: Independencia, Humay, El Carmen y Chincha Baja, entre ju- nio y agosto de 2017. Los distritos de Humay e Independencia pertenecen a la provincia de Pisco y se encuentra a una altitud de 410 y $211 \mathrm{msnm}$, respectivamente (INEI, 2014). Los distritos de El Carmen y Chincha Baja pertenecen a la provincia de Chincha y están ubicadas a una altitud de 153 y $33 \mathrm{msnm}$, respectivamente (INEI, 2014). Los cuatro distritos presentan un clima muy similar, son de tipo árido, semicálido, con escasas precipitaciones anuales (verano: de $2.98 \mathrm{~mm}$; invierno: $0.28 \mathrm{~mm}$ ). La humedad relativa promedio es de $75 \%$ y la temperatura oscila entre $9{ }^{\circ} \mathrm{C}$ en invierno a $32.9^{\circ} \mathrm{C}$ en verano, con un promedio de $25^{\circ} \mathrm{C}$ (SENAMHI, 2018).

\section{Población y Muestra}

La población caprina varió entre 1489 a 7906 cabezas por distrito (INEI, 2012). Se contó con la colaboración de las cuatro municipalidades en cumplimiento de la Meta 37 dada por el SENASA (Implementación de las acciones de prevención y control sanitario de enfermedades parasitarias de los animales en el ámbito de su jurisdicción), además del apoyo técnico del Laboratorio de Parasitología de la Facultad de Medicina Veterinaria (FMV), Universidad Nacional Mayor de San Marcos (UNMSM), Lima.

Para el cálculo del tamaño de muestra se usó la fórmula de proporciones finitas (Daniel, 2007). Se consideró la población total de 72112 animales, según el IV Censo Nacional Agropecuario (2012), una prevalencia esperada de 69\% (Casas et al., 1993), un nivel de confianza del $95 \%$ y un error del $5 \%$ obteniéndose una cantidad mínima a muestrear de 327 cabras. Para determinar el número de muestras por distrito se usó la fórmula de estratificación de Pérez (2000), resultando cantidades mínimas de animales por distrito: Independencia (137), Humay (99), El Carmen (66) y Chincha Baja (26); sin embargo, gracias al apoyo de los productores se pudo colectar muestras de 728 cabras, siendo $240,130,88$ y 270 cabras de cada distrito, respectivamente. 
Se muestrearon caprinos aparentemente saludables y sin diferencia por sexo. Cómo criterio de inclusión solo se consideró a los animales que no hayan sido desparasitados en los últimos tres meses y como criterio de exclusión a los animales menores de seis meses y hembras gestantes.

\section{Toma de Muestras}

Las muestras de heces se tomaron directamente del recto y fueron almacenadas en un contenedor térmico con geles refrigerantes $\left(4-8{ }^{\circ} \mathrm{C}\right)$ (Rojas, 2004) para su inmediato traslado al Laboratorio de Parasitología de la FMV-UNMSM para su procesamiento.

\section{Diagnóstico Coproparasitológico}

La presencia y los tipos de huevos de nematodos se determinaron mediante la técnica cualitativa de flotación con solución de Sheather. La carga parasitaria (número de huevos de nematodos por gramo de heces hpg) se obtuvo mediante la técnica cuantitativa de McMaster modificado (Rojas, 2004).

\section{Cultivo e Identificación de Nematodos}

Las muestras de heces positivas a huevos tipo Strongylus (HTS) fueron sometidas a cultivo de larvas por la técnica de Corticelli y Lai y las larvas infectivas (L3) fueron recuperadas por el método de Baerman (Rojas, 2004). Los géneros de las larvas infectivas (L3) fueron determinados mediante claves taxonómicas según sus características morfológicas y biométricas (Levine, 1968; Ueno y Gonçalves, 1998; Van Wyk et al., 2004).

\section{Análisis de la Información}

Se calculó la prevalencia de nematodos mediante la determinación del número de muestras fecales positivas para cada parásito expresándose en forma porcentual. La probable asociación entre la prevalencia general de nematodos con las variables procedencia (Independencia, Humay, El Carmen y Chincha Baja), edad ( $<1,1-<3, \geq 3$ años) y sexo (hembra y macho) fue determinada mediante la prueba de Chi cuadrado (Daniel, 2007).

Se determinó la prevalencia de cada tipo de huevo de nematodo (HTS, Skrjabinema spp y Trichuris spp) y se evaluó la posible asociación de cada uno con las variables de interés (procedencia, edad y sexo) mediante la prueba de Chi cuadrado (Daniel, 2007). La carga parasitaria (hpg) de los tipos de huevos se expresó en función a su media geométrica (MG). El análisis de la relación entre la carga parasitaria y las variables de estudio se realizó mediante las pruebas no paramétricas de U-Mann Whitney para el caso de sexo y Kruskall Wallis para procedencia y edad (Daniel, 2007). Además, se determinó la prevalencia por género / especies de lavas infectivas L3 de los huevos HTS cultivados. Los datos se procesaron con el paquete de análisis estadístico Stata IC 15 (Stata Corp, College Station TX).

\section{Resultados}

La prevalencia general de nematodos gastrointestinales en muestras fecales de caprinos pertenecientes a cuatro distritos de la región Ica fue 67.6\% (IC 95\%: 64.1-70.9). Las prevalencias según distritos fueron: Independencia $92.5 \%$, Humay $80.8 \%$, El Carmen 59.1\% y Chincha Baja 41.9\% (Cuadro 1). La prueba de Chi cuadrado demostró asociación significativa entre la prevalencia de nematodos con todas las variables de estudio; así la prevalencia fue mayor en caprinos de $1-<3$ años $(74.5 \%)$ y en hembras $(68.6 \%)$ (Cuadro 1).

Con respecto a los tipos de huevos de nematodos detectados, el $63.7 \%$ de los caprinos presentaron huevo tipo Strongylus (HTS), mientras que 12.9 y $4.1 \%$ mostraron huevos compatibles con los géneros Skrja- 
Cuadro 1. Prevalencia general (\%) de nematodos gastrointestinales y su asociación con las variables edad, sexo y procedencia en caprinos de cuatro distritos de Ica (2017)

\begin{tabular}{lccccc}
\hline \multirow{2}{*}{ Variable } & \multicolumn{3}{c}{ Animales } & \multicolumn{2}{c}{ Prevalencia } \\
\cline { 2 - 6 } & $\begin{array}{c}\text { Muestreados } \\
(\mathrm{n})\end{array}$ & $\begin{array}{c}\text { Positivos } \\
(\mathrm{n})\end{array}$ & $\%$ & IC 95\% & $p^{a}$ \\
\hline Edad (años) & 30 & 10 & 33.3 & $18.3-52.8$ & $<0.001$ \\
$\quad<1$ & 552 & 411 & 74.5 & $70.6-77.9$ & \\
$\quad 1 \mathrm{a}<3$ & 146 & 71 & 48.6 & $40.5-56.8$ & \\
$\quad \geq 3$ & & & & & \\
Sexo & 707 & 485 & 68.6 & $65.1-71.9$ & 0.001 \\
$\quad$ Hembra & 21 & 7 & 33.3 & $15.7-57.4$ & \\
$\quad$ Macho & & & & & \\
Procedencia & 240 & 222 & 92.5 & $88.4-95.2$ & $<0.001$ \\
$\quad$ Independencia & 130 & 105 & 80.8 & $72.9-86.7$ & \\
$\quad$ Humay & 88 & 52 & 59.1 & $48.4-69.0$ & \\
$\quad$ El Carmen & 270 & 113 & 41.9 & $36.1-47.9$ & \\
$\quad$ Chincha Baja & 728 & 492 & 67.6 & $64.1-70.9$ & \\
\hline Total & & & &
\end{tabular}

a Prueba de chi cuadrado

binema y Trichuris, respectivamente (Cuadro 2). La mayor prevalencia de huevos HTS se observó en los caprinos de $1-<3$ años $(71 \%)$, en hembras $(64.8 \%)$ y en los animales del distrito de Independencia (90.8\%) (Cuadro 2).

La prevalencia de huevos del género Skrjabinema fue mayor en caprinos de 1$<3$ años (15.3\%), en hembras (13.2\%) y en los distritos de Independencia (17.6\%) y Humay (16.3\%). demostrándose asociación significativa entre la prevalencia de estos huevos con las variables edad ( $\mathrm{p}=0.002$ ) y procedencia $(\mathrm{p}=0.001)$. La prevalencia de huevos del género Trichuris fue muy baja (4.1\%) y sin diferencias por efecto de la edad, procedencia y sexo de los animales (Cuadro 2).
La carga parasitaria general de HTS mostró una media geométrica de $259.4 \mathrm{hpg}$. Los animales de 1-<3 años, las hembras y los procedentes del distrito de Independencia presentaron las mayores cargas parasitarias con medias geométricas de hpg de 319.3, 264.6 y 579.9 , respectivamente. Asimismo, se halló asociación entre la carga parasitaria de huevos HTS con todas las variables (Cuadro 3 ).

La media geométrica de la carga parasitaria general de los huevos de Skrjabinema spp fue 70 hpg y de los huevos de Trichuris spp de $54.3 \mathrm{hpg}$, sin hallarse diferencias significativas por efecto de las variables en estudio. Cabe señalar que la asociación entre la carga parasitaria de los huevos de Trichuris 
Cuadro 2. Prevalencia (\%) de tipos de huevos de nematodos y su asociación con las variables edad, sexo y procedencia en caprinos de cuatro distritos de Ica (2017)

\begin{tabular}{|c|c|c|c|c|c|c|c|c|c|}
\hline \multirow[t]{2}{*}{ Variable } & \multicolumn{3}{|c|}{ HTS } & \multicolumn{3}{|c|}{ Skrjabinema spp } & \multicolumn{3}{|c|}{ Trichuris spp } \\
\hline & $\mathrm{n}$ & $\%$ & $p^{a}$ & $\mathrm{n}$ & $\%$ & $p$ & $\mathrm{n}$ & $\%$ & $p^{b}$ \\
\hline \multicolumn{10}{|l|}{ Edad(años) } \\
\hline$<1$ & 7 & 23.3 & $<0.001$ & 4 & 13.3 & $0.002^{\mathrm{a}}$ & 0 & 0.0 & 0.711 \\
\hline $1 \mathrm{a}<3$ & 392 & 71.0 & & 84 & 15.3 & & 23 & 4.2 & \\
\hline$\geq 3$ & 65 & 44.5 & & 6 & 4.1 & & 7 & 4.8 & \\
\hline \multicolumn{10}{|l|}{ Sexo } \\
\hline Hembra & 458 & 64.8 & 0.001 & 93 & 13.2 & $0.220^{\mathrm{b}}$ & 30 & 4.2 & 0.408 \\
\hline Macho & 6 & 28.6 & & 1 & 4.8 & & 0 & 0.0 & \\
\hline \multicolumn{10}{|l|}{ Procedencia } \\
\hline Independencia & 218 & 90.8 & $<0.001$ & 42 & 17.6 & $0.001^{\mathrm{a}}$ & 14 & 5.8 & 0.186 \\
\hline Humay & 103 & 79.2 & & 21 & 16.3 & & 6 & 4.6 & \\
\hline El Carmen & 51 & 57.9 & & 2 & 2.3 & & 4 & 4.6 & \\
\hline Chincha Baja & 92 & 34.1 & & 29 & 10.7 & & 6 & 2.2 & \\
\hline $\begin{array}{l}\text { Total } \\
\text { (IC 95\%) }\end{array}$ & 464 & $(60$. & $\begin{array}{l}3.7 \\
-67.2)\end{array}$ & 94 & (10. & $\begin{array}{l}9 \\
15.6)\end{array}$ & 30 & & $\begin{array}{l}.1 \\
-5.8)\end{array}$ \\
\hline
\end{tabular}

a Prueba de chi cuadrado; ${ }^{b}$ Prueba exacta de Fisher

spp y el sexo no pudo ser evaluada debido a que ningún animal macho resultó positivo a este tipo de parásito (Cuadro 3).

El cultivo larvas de muestras de muestras con altas cargas de huevos HTS resultó con las siguientes prevalencias: Haemonchus spp (45\%), Trichostrongylus spp (37\%), Ostertagia spp (15\%), Chabertia ovina (0.6\%) y Cooperia spp (0.6\%) (Cuadro 4).

\section{Discusión}

Se encontró una alta prevalencia general (67.6\%) de nematodos gastrointestinales en las cabras del estudio; resultado concordante con los estudios previos en el país (Torres, 1952; Casas, 1993). Estudios precedentes llevados a cabo en otros países indican que la alta prevalencia puede estar muy relacionada al tipo de crianza, siendo más prevalente en explotaciones extensivas, debido al inadecuado control sanitario y a las condiciones climáticas y geográficas de la zona (Cordero del Campillo et al., 1999; Quiroz, 2008; Muluneh et al., 2014). Así, en África tropical, Agyei y Amponsah (2001) reportaron cerca del $95 \%$ positividad a nematodos en ovejas y cabras de crianza extensiva, en tanto que en Venezuela se halló $65 \%$ de prevalencia (Quijada et al., 2012) y en Brasil de 91.7\% (Martins-Filho y Menezes, 2001).

La crianza extensiva, al estar basada en el pastoreo conlleva a una mayor probabilidad de ingerir larvas infectivas (L3) presentes en pasturas contaminadas, lo cual unido a la no dosificación empleo de suplementos nutricionales conllevan a una alta morbilidad, tal y como se ha encontrado en el presente estudio (Cordero del Camillo et al., 1999; Quiroz, 2008). 
Cuadro 3. Media geométrica (MG) de la carga parasitaria (hpg) de huevos tipo Strongylus (HTS), Skrjabinema spp y Trichuris spp presentes en caprinos positivos de cuatro distritos de Ica (2017)

\begin{tabular}{|c|c|c|c|c|c|c|}
\hline \multirow[b]{2}{*}{ Variables } & \multicolumn{2}{|l|}{ HTS } & \multicolumn{2}{|c|}{ Skrjabinema spp } & \multicolumn{2}{|c|}{ Trichuris spp } \\
\hline & $\begin{array}{l}\text { MG (IC } \\
95 \%)\end{array}$ & $p$ & $\begin{array}{l}\text { MG (IC } \\
95 \%)\end{array}$ & $p$ & $\begin{array}{l}\text { MG (IC } \\
95 \%)\end{array}$ & $p$ \\
\hline \multicolumn{7}{|l|}{ Edad (años) } \\
\hline$<1$ año & $\begin{array}{c}55.2 \\
(43.3-70.3)\end{array}$ & $0.0001^{\mathrm{a}}$ & $\begin{array}{c}50.0 \\
(50.0-50.0)\end{array}$ & $0.4110^{\mathrm{a}}$ & 0 & $0.3226^{\mathrm{a}}$ \\
\hline $1 \mathrm{a}<3$ años & $\begin{array}{c}319.3 \\
(275.0-370.7)\end{array}$ & & $\begin{array}{c}69.5 \\
(60.3-80.1)\end{array}$ & & $\begin{array}{c}55.7 \\
(49.1-63.3)\end{array}$ & \\
\hline$\geq 3$ años & $\begin{array}{c}87.6 \\
(72.6-105.6)\end{array}$ & & $\begin{array}{c}96.6 \\
(30.9-302.0)\end{array}$ & & $\begin{array}{c}50.0 \\
(50.0-50.0)\end{array}$ & \\
\hline \multicolumn{7}{|l|}{ Sexo } \\
\hline Hembras & $\begin{array}{c}264.6 \\
(230.8-303.5)\end{array}$ & $0.0055^{\mathrm{b}}$ & $\begin{array}{c}70.2 \\
(61.1-80.8)\end{array}$ & $0.5740^{\mathrm{b}}$ & $\begin{array}{c}54.3 \\
(49.3-59.9)\end{array}$ & - \\
\hline Machos & $\begin{array}{c}56.1 \\
(41.7-75.5)\end{array}$ & & 50 & & 0 & \\
\hline \multicolumn{7}{|l|}{ Procedencia } \\
\hline Independencia & $\begin{array}{c}579.9 \\
(476.3-705.9)\end{array}$ & $0.0001^{\mathrm{a}}$ & $\begin{array}{c}66.3 \\
(54.8-80.2)\end{array}$ & $0.6144^{\mathrm{a}}$ & $\begin{array}{c}56.8 \\
(46.8-68.9)\end{array}$ & $0.6552^{\mathrm{a}}$ \\
\hline Humay & $\begin{array}{c}193.8 \\
(152.1-246.8)\end{array}$ & & $\begin{array}{c}75.3 \\
(53.1-106.8)\end{array}$ & & $\begin{array}{c}56.1 \\
(41.7-75.5)\end{array}$ & \\
\hline El Carmen & $\begin{array}{c}86.5 \\
(70.5-106.0)\end{array}$ & & $\begin{array}{c}180.3 \\
(0-2.2)\end{array}$ & & $\begin{array}{c}50.0 \\
(50.0-50.0)\end{array}$ & \\
\hline Chincha Baja & $\begin{array}{c}98.3 \\
(80.9-119.3)\end{array}$ & & $\begin{array}{c}67.2 \\
(53.5-84.5)\end{array}$ & & $\begin{array}{c}50.0 \\
(50.0-50.0)\end{array}$ & \\
\hline $\begin{array}{l}\text { Total } \\
\text { (IC 95\%) }\end{array}$ & $\begin{array}{c}259.4 \\
(226.4-297.2)\end{array}$ & & $\begin{array}{c}70.0 \\
(60.9-80.4)\end{array}$ & & $\begin{array}{c}54.3 \\
(49.3-59.9)\end{array}$ & \\
\hline
\end{tabular}

a Prueba de Kruskall Wallis; ${ }^{b}$ Prueba de U-Mann Whitney; - No evaluado

En relación con el control sanitario, se observó que los productores carecían de un calendario sanitario y solo vacunaban contra brucelosis por medio de las campañas del SENASA (Servicio Nacional de Sanidad Agraria del Perú). La dosificación antiparasitaria era realizada a criterio propio, sin orientación técnica y en su mayoría por conveniencia, variando entre 3 a 12 meses. Asimismo, gran parte de los ganaderos despara- sitaban con albendazol; el cual, es un producto que ha venido siendo utilizado desde hace muchos años ocasionando resistencia, principalmente en nematodos de los géneros Haemonchus y Trichostrongylus (Fiel et al., 2000; Estrada, 2010; Arece et al., 2016). Este tipo de manejo sanitario, llevado a cabo por mucho tiempo en el país, explicaría la alta prevalencia encontrada. 
Cuadro 4. Prevalencia (\%) de géneros y especies de larvas infectivas (L3) obtenidas de muestras de heces con altas cargas de HTS, en caprinos de cuatro distritos de Ica (2017)

\begin{tabular}{lc}
\hline $\begin{array}{c}\text { Géneros / especies de } \\
\text { larvas (L3) }\end{array}$ & $\begin{array}{c}\text { Prevalencia } \\
(\%)\end{array}$ \\
\hline Haemonchus spp & 45 \\
Trichostrongylus spp & 37 \\
Ostertagia spp & 15 \\
Chabertia ovina & 0.6 \\
Cooperia spp & 0.6 \\
\hline
\end{tabular}

Los distritos de Independencia y Humay presentaron mayores prevalencias $(92.5 \mathrm{y}$ $80.8 \%$, respectivamente) en comparación con los distritos de El Carmen (59.1\%) y Chincha Baja $(41.9 \%)(p<0.001)$. Estos resultados estarían probablemente relacionados con las condiciones ambientales y geológicas favorables en estos distritos que propician el desarrollo del parasitismo, así como al manejo deficiente de los antihelmínticos. Si bien la precipitación pluvial es escasa en las zonas del estudio, Humay e Independencia poseen suelos con alta humedad debido a la presencia de zonas pantanosas con flujos de agua provenientes de pozos subterráneos, así como riachuelos y acequias originadas del rio Pisco (SENAMHI, 2018). Esta humedad constante cerca de los sembríos y pampas hace que sea favorable la supervivencia de los huevos y el desarrollo de larvas infectivas (L3) en las pasturas permitiendo la continuidad del ciclo biológico en forma permanente e incrementando la probabilidad de su consumo durante el pastoreo (Urquhart et al., 2001; Barriga, 2002; Quiroz, 2008).

Los caprinos de $1-<3$ años presentaron una mayor prevalencia (74.5\%) en comparación con los menores de 1 año $(33.3 \%)$ y los mayores a 3 años $(48.6 \%)(\mathrm{p}<0.001)$, po- siblemente debido a que la mayoría de los animales estaba cerca del año de edad y, por lo tanto, mostraban una insuficiente respuesta inmune contra infecciones parasitarias. Vale decir que se ha determinado que la respuesta inmune eficiente se logra en cabritos entre los 12 y los 18 meses de edad (Vlassoff et al., 1999). De otro lado, la baja prevalencia hallada en cabritos menores a un año se deba probablemente al escaso número de animales evaluados $(n=30)$, esto debido a la renuencia de los propietarios de permitir que los animales más pequeños sean muestreados. Asimismo, se observó que los animales más jóvenes se mantenían en estabulación, de allí que la probabilidad de contagio haya sido menor (Castillo, 2003).

La mayor prevalencia en hembras (68.6\%) que en machos (33.3\%) (Cuadro 1; $\mathrm{p}=0.001$ ) pudo deberse igualmente a la menor población de machos en los criaderos $(n=21)$, toda vez que los productores solo necesitan tener un macho reproductor por cada 100 hembras, y el resto es vendido a partir del mes de edad debido a la alta demanda de su carne. De esta forma, la mayoría de machos muestreados eran adultos $(76 \%) \mathrm{y}$, por lo tanto, con un completo desarrollo de su sistema inmune (Vlassoff et al., 1999).

Respecto al tipo de huevos (Cuadro 2), se halló una alta prevalencia (63.7\%) de huevos tipo Strongylus (HTS), en comparación con los de Skrjabinema (12.9\%) y Trichuris (4.1\%). La mayor prevalencia general de huevos HTS probablemente fue influenciada por las condiciones climáticas y geológicas (Quiroz, 2008; Taylor et al., 2016). Respecto a los huevos oxiuroideos, específicamente los del género Skrjabinema suelen encontrarse con frecuencia en caprinos, siendo de baja patogenicidad (Quiroz, 2008; Zajac y Conboy, 2012). Por otro lado, la prevalencia de huevos del género Trichuris suele ser baja en condiciones de crianza extensiva (Rossanigo, 2007). 
Ninguna de las medias geométricas de las cargas parasitarias halladas fue alta (Cuadro 3). Solo en el distrito de Independencia (579.9 hpg) y en los animales de $1-<3$ años (319.3 hpg) se encontraron cargas moderadas (300700 hpg) (Skerman y Hillard, 1966). La carga parasitaria está relacionada al potencial biótico y a la hipobiosis de cada nematodo siendo afectadas principalmente por las condiciones medioambientales de la época de muestreo (Rojas, 2004; Ghalsasi et al., 2015).

Las hembras de los nematodos con HTS poseen un alto potencial biótico, así Haemonchus spp puede ovopositar de 500015000 huevos/día, Trichostrongylus spp de 100-200 huevo/día (Ueno y Gonçalves, 1998; Cordero y Salas, 2000; Arece et al., 2016), Chabertia ovina de 3000 huevos/día (Martínez, 2008), Ostertagia spp de 200-300 huevos/día y Cooperia spp 1000 huevos/día; de igual manera sucede con Skrjabinema spp y Trichuris spp con 3000-10 000 huevos/día (Cordero y Salas, 2000). No obstante, la carga parasitaria hallada fue en su mayoría baja y esto debido principalmente a que el muestreo se realizó entre junio y agosto, meses de época seca. Si bien, el pastoreo, la humedad del suelos, deficiencias nutricionales, baja respuesta inmunológica y la resistencia antihelmíntica favorecen la diversidad de especies parasitarias y explican las asociaciones halladas, la escasa precipitación $(0.28 \mathrm{~mm})$ y la baja temperatura (mínimo de $9^{\circ} \mathrm{C}$ en julio) (SENAMHI, 2018) que se da en los meses de invierno influyen negativamente en el potencial biótico, reduciendo el metabolismo de los nematodos con huevos tipo HTS, no permitiendo su reactivación, ya que este evento necesita de temperaturas constantes mayores a $15^{\circ} \mathrm{C}$ (Rojas, 2004; Ghalsasi et al., 2015).

Los géneros más frecuentes de larvas (L3) infectivas halladas en el presente estudio fueron Haemonchus (45\%) y Trichostrongylus (37\%), resultados similares a los encontrados por Gonzales (1968) y Casas (1993) en Ica y Carruitero (1966) en Lima. Cabe resaltar que dichos estudios em- plearon la técnica de recuento diferencial de parásitos al examen post mortem; el cual es el método más sensible para detectar las especies parasitarias presentes en animales (Rojas, 2004).

Por otro lado, estudios más actuales en otros países reportaron la presencia de estas mismas especies por el tipo de clima templado-cálido. Estudios en Antioquia, Colombia, reportaron una alta prevalencia de Trichostrongilidos, siendo Haemonchus contortus (61.3\%), Ostertagia circumcincta (25.5\%) y Trichostrongylus spp (21.5\%) los parásitos más frecuentes (Zapata et al., 2016). Así mismo, estudios realizados en Argentina reportaron un predominio de Haemonchus spp (73.5\%) en primavera y de Trichostrongylus spp (40.5\%) (Suarez et al., 2013). De otra parte, en Huambo, Angola se reportaron cinco géneros de estrongilidos gastrointestinales en caprinos (Haemonchus spp, Oesophagopstomun spp, Trichostrongylus spp, Ostertagia spp y Cooperia spp), siendo Haemonchus el género predominante (Paixão et al., 2015)

\section{Conclusiones}

- La prevalencia general de nematodos gastrointestinales en caprinos de cuatro distritos del departamento de Ica fue de $67.6 \%$.

- Se encontró asociación entre la prevalencia de nematodos gastrointestinales con las variables edad, sexo y procedencia $(\mathrm{p}<0.05)$, siendo mayor en los caprinos de los distritos de Independencia (92.5\%) y Humay $(80.8 \%)$, en las hembras $(68.6 \%)$ y en animales de $1-<3$ años $(74.5 \%)$.

- $\quad$ El $63.7 \%$ de los caprinos presentaron huevo tipo Strongylus (HTS) y solo el $12.9 \%$ y $4.1 \%$ presentaron huevos compatibles con los géneros Skrjabinema y Trichuris, respectivamente. Siendo las cargas consideradas como bajas en todos los casos. 
- Los principales géneros y especies de larvas infectivas (L3) fueron Haemonchus spp (45\%), Trichostrongylus spp (37\%), Ostertagia spp (15\%), Chabertia ovina $(0.6 \%)$ y Cooperia spp. $(0.6 \%)$.

\section{Literatura Citada}

1. Agyei AD, Amponsah A. 2001. Seasonal availability of infective strongylate nematode larvae on pasture in the forest zone of Ghana. Bull Anim Health Prod Afr 49: 68-72.

2. Arroyo O. 2007. Situación actual y proyecciones de la crianza de caprinos en el Perú. En: XX Reunión ALPA. Cusco, Perú: Asociación Latinoamericana de Producción Animal.

3. Arece J, López Y, Flores UD, Ramón D, Macedo E, Rojo R. 2016. Diagnóstico de resistencia al albendazol sulfóxido en ovejas y cabras, en la provincia de Matanzas. Cuba. Pastos y Forrajes 39: 286-290.

4. Barriga O. 2002. Enfermedades parasitarias de los animales domésticos en la América Latina. Santiago: Germinal. $247 \mathrm{p}$.

5. Boyazoglu J, Hatziminaoglou I, Morand-Fehr P. 2005. The role of the goat in society: past, present and perspectives for the future. Small Ruminant Res 60: 13-23. doi: 10.1016/ j.smallrumres.2005.06.003

6. Carruitero Z. 1966. Encuesta de infestación nematídica en caprinos en el departamento de Lima. Tesis de Médico Veterinario. Lima, Perú: Univ. Nacional Mayor de San Marcos. 37 p.

7. Casas E. 1993. Prevalencia de helmintos gastrointestinales en ganado caprino beneficiado en la provincia de $\mathrm{Ca}-$ ñete. Tesis de Médico Veterinario. Lima, Perú: Univ. Nacional Mayor de San Marcos. 50 p.
8. Castillo M. 2003. Estudio epidemiológico de parasitismo gastrointestinal en caprinos lecheros en comuna de Purranque, Décima región de Los Lagos, Chile: periodo otoño-invierno. Tesis de Médico Veterinario. Universidad Austral de Chile. $42 \mathrm{p}$.

9. Chávez GC, Guerrero DC. 1960. Ecto y endoparásitos identificados en el departamento de parasitología de la Facultad de Medicina Veterinaria (1947-1960). Rev Cient-Fac Cien V 15: 48-68.

10. Cordero UL, Salas JJ. 2000. Enfermedades de los animales domésticos. San José: EUNED. 216 p.

11. Cordero del Campillo M, Rojo FD, Martínez AR, Sánchez MC, Hernández S, Navarrete I, Diez P, et al. 1999. Parasitología veterinaria. Madrid, España: McGraw Hill Interamericana. $968 \mathrm{p}$.

12. Daniel W. 2007. Bioestadística: bases para el análisis de las ciencias de la salud. $4^{\mathrm{a}}$ ed. México: Limusa. 924 p.

13. Estrada I. 2010. Determinación de resistencia a helminticidas en cabras de Nueva Esperanza, Sololá, Guatemala. Tesis de Médico Veterinario. Guatemala: Univ. de San Carlos de Guatemala. 92 p.

14. Fiel C, Hansen M, Lizziero M, Saumel C, Steffan P, Fuse L, Lutzelschwab C. 2000. Resistencia antihelmíntica en cabras lecheras. En: III Congreso Argentino de Parasitología. Buenos Aires.

15. Flores MA, Pérez $R$, Basurto $M$, Jurado $M R$. 2009. La leche de cabra y su importancia en la nutrición. Tecnociencia Chihuahua 3: 107-113.

16. Ghalsasi PM, Ghalsasi PP, Bandgar RS, Nimbkar C. 2015. Indications of hypobiosis of Haemonchus contortus in goats od deccan plateau in Maharashtra. Ind J Small Ruminants 21: 354-355. doi: 10.5958/0973-9718.2015.00083.5 
17. Gonzales Z. 1968. Encuesta de parásitos gastrointestinales en caprinos procedentes del departamento de Ica. Tesis de Médico Veterinario. Ica, Perú: Univ. Nacional San Luis Gonzaga de Ica. 69 p.

18. Hassan MM, Hoque MA, Islam $S K M A$, Khan $S A$, Roy K, Banu $Q$. 2011. A prevalence of parasites in Black Bengal goats in Chittagong, Bangladesh. Inter J Livestock Prod 2: 40-44.

19. [INEI] Instituto Nacional de Estadística e Informática. 2012. IV Censo Nacional Agropecuario. Lima. [Internet]. Disponible en: http://siea.minag.gob.pe/ siea/?q=datos-iv-cenagro-2012

20. [INEI] Instituto Nacional de Estadística e Informática. 2014. Población total proyectada y ubicación geográfica de la capital legal según provincia y distrito. Lima. [Internet]. Disponible en: https://www.inei.gob.pe/media/ MenuRecursivo/publicaciones.../Est/.../ ica_11_3.xls

21. Levine ND. 1968. Nematoda parasites of domestic animals and man. USA: Burguess Publ. 600 p.

22. Martínez AJ. 2008. Comparación de la presencia de fases larvarias de nematodos gastrointestinales en bovinos, en sistemas silvopastoriles y no silvopastoriles en el Municipio de San Andrés Villa Seca, Retalhuleu. Tesis de Médico Veterinario Zootecnista. Guatemala: Univ. de San Carlos de Guatemala. 47 p.

23. Martins-Filho E, Menezes RCAA. 2001. Parasitos gastrintestinais em caprinos (Capra hircus) de uma criação extensiva na microrregião de Curimataú, estado da Paraíba, Brasil. Rev Bras Parasitol Vet 10: 41-44.

24. [MIDAGRI] Ministerio de Agricultura y Riego. 2015. [Internet]. Disponible en: https://www.midagri.gob.pe/portal/40-sector-agrario/situacion-de-lasactividades-de-crianza-y-producci/299caprinos

25. Muluneh J, Bogale B, Chanie M. 2014. Major gastrointestinal nematodes of small ruminants in Dembia district, northwest Ethiopia. Eur J Appl Sci 6: 30 36. doi: 10.5829/idosi.ejas.2014.6.2.8652

26. Nolte E. 1979. Bibliografía peruana sobre caprinos. Lima, Perú: Univ. Nacional Agraria La Molina. $75 \mathrm{p}$.

27. Odogu KI, Okaka CE. 2016. Prevalence of gastrointestinal parasites of goats slaughtered at Aduwawa Abattior, Benin City, Edo State, Nigeria. Int J Innovative Agric Bio Res 4: 22-26.

28. Paixã̃o A, Walter A, Esperança $S$, de Fontes-Pereira M, Ataulfo M, Jamba J, Sánchez L, et al. 2015. Identification of the genera Haemonchus, Trichostrongylus, Oesophagostomum, Ostertagia and Cooperia in goats in the province of Huambo-Angola. Rev Salud Anim 37: 64-68

29. Park Y, Haenlein G. 2006. Manual de la leche de los mamíferos no bovinos. Zaragoza, España: Acribia. $456 \mathrm{p}$

30. Pérez C. 2000. Técnicas de muestreo estadístico. México: Alfa Omega. 603 p.

31. Quijada J, Bethencourt A, Sulbarán D, Salcedo P, Aguirre A, Vivas I, López E, et al. 2012. Estrongilidos digestivos en caprinos: contajes fecales de huevos y valores de la escala Famacha ${ }^{\circledR}$ en un rebaño infectado naturalmente. Rev Cient-Fac Cien V 22: 418-425.

32. Quiroz H. 2008. Parasitología y enfermedades parasitarias de animales domésticos. México DF: Limusa. 876 p.

33. Rojas M. 2004. Nosoparasitosis de los rumiantes domésticos peruanos. $2^{\mathrm{a}} \mathrm{ed}$. Lima: Martegraf. $154 \mathrm{p}$.

34. Rossanigo C. 2007. Parasitosis de las cabras. En: Enfermedades parasitarias de los ovinos y otros rumiantes menores en el cono sur de América. Publicación Técnica $\mathrm{N}^{\circ}$ 70. INTA - EEA Anguil 247252 p. [Internet]. Disponible en: https:// inta.gob.ar/sites/default/files/script-tmppubli70_-_ver_editores_y_autores_colaboradores.pdf

35. [SENAMHI] Servicio Nacional de Meteorología e Hidrología del Perú. 2018. Lima. [Internet]. Disponible en: https://www.senamhi.gob.pe/?p=-pronostico detalle-turistico-\&localidad $=0029$ 
36. Skerman K, Hillard J. 1966. A handbook for studies of helminth parasites of ruminants. Teheran: Near East Animal Health Institutes, Iran Unit, United Nations Development Program/Special Fund, Executing Agency FAO of the United States. 200 p.

37. Suarez V, Fondraz M, Viñabal A, Martínez G, Salatin A. 2013. Epidemiología de los nematodes gastrointestinales en caprinos lecheros en los valles templados del NOA, Argentina. Rev Invest Agropec 39: 191-197. doi: https://doi.org/10.4102/ojvr.v84i1.1240

38. Sukupayo P, Rayamajhee B. 2018. Prevalence of intestinal helminth parasites in goats of Koshi rural munucipality, Nepal. IJSER 9: 1471-1475.

39. Taylor MA, Coop RL, Wall RL. 2016. Veterinary parasitology. $3^{\text {rd }}$ ed. Oxford: Blackwell Publising. $600 \mathrm{p}$.

40. Torres A. 1952. Survey del parasitismo gastrointestinal en caprinos procedentes de la provincia de Yauyos departamento de Lima. Tesis de Médico Veterinario. Lima, Perú: Univ. Nacional Mayor de San Marcos. 56 p.

41. Ueno H, Gonçalves P. 1998. Manual para diagnóstico de helmintos de rumiantes. $4^{\mathrm{a}}$ ed. Tokyo: Japan International Cooperatoin Agency. 143. Disponible en: https://rl.ufrrj.br/adivaldofonseca/wpcontent/uploads/2014/06/manual_helmintoses-UENO-site-do-CBPV.pdf
42. Urquhart G, Armour J, Dundan J, Dunn A, Jennings F. 2001. Parasitología veterinaria. $2^{a}$ ed. Acribia. $355 \mathrm{p}$.

43. Van Wyk J, Cabaret J, Michael L. 2004. Morphological identification of nematode larvae of small ruminants and cattle simplified. Vet Parasitol 119: 277306. doi: 10.1016/j.vetpar.2003.11.012

44. Vlassoff A, Bissett S, McMurty L. 1999. Faecal egg counts in Angora goats following natural or experimental challenge with nematode parasites: within flock variabilities and repeatabilities. Vet Parasitol 84: 113-123. doi: 10.1016/ s0304-4017(99)00072-2

45. Zajac A, Conboy G 2012. Veterinary clinical parasitology. $8^{\text {th }}$ ed. Iowa: WileyBlackwell. 368 p.

46. Zapata SR, Velásquez VR, Herrera OL, Ríos OL, Polanco ED. 2016. Prevalencia de nematodos gastrointestinales en sistemas de producción ovina y caprina bajo confinamiento, semiconfinamiento y pastoreo en municipios de Antioquia, Colombia. Rev Inv Vet Perú 27: 344-354. doi: 10.15381/rivep.v27i2.11647

47. Zárate D, Rojas J, Segura A. 2017. Validación del método FAMACHAC para dosificación antihelmíntica selectiva en rebaños caprinos lecheros. Rev Inv Vet Perú 28: 150-159. doi: 10.15381/ rivep.v28i1.12934 